\title{
Reconciling marginalism with egalitarianism: consistency, monotonicity, and implementation of egalitarian Shapley values
}

\author{
René van den Brink • Yukihiko Funaki • Yuan Ju
}

Received: 20 May 2010 / Accepted: 3 December 2011 / Published online: 22 December 2011 C The Author(s) 2011. This article is published with open access at Springerlink.com

\begin{abstract}
One of the main issues in economic allocation problems is the trade-off between marginalism and egalitarianism. In the context of cooperative games this trade-off can be framed as one of choosing to allocate according to the Shapley value or the equal division solution. In this paper we provide three different characterizations of egalitarian Shapley values being convex combinations of the Shapley value and the equal division solution. First, from the perspective of a variable player set, we show that all these solutions satisfy the same reduced game consistency. Second, on a fixed player set, we characterize this class of solutions using monotonicity properties. Finally, towards a strategic foundation, we provide a non-cooperative implementation for these solutions which only differ in the probability of breakdown at a certain stage of the game. These characterizations discover fundamental differences as well as intriguing connections between marginalism and egalitarianism.
\end{abstract}

\footnotetext{
R. van den Brink $(\varangle)$

Department of Econometrics and Tinbergen Institute, VU University,

De Boelelaan 1105, 1081 HV Amsterdam, The Netherlands

e-mail: jrbrink@feweb.vu.nl

Y. Funaki

Department of Economics, School of Political Science and Economics, Waseda University, 1-6-1 Nishi-Waseda, Shinjuku-Ku, Tokyo 169-8050, Japan e-mail: funaki@waseda.jp

Y. Ju

Department of Economics and Related Studies, University of York, Heslington, York YO10 5DD, UK e-mail: yuan.ju@york.ac.uk
} 


\section{Introduction}

One of the main issues in economic allocation problems is the trade-off between marginalism and egalitarianism. In this paper we consider this issue in the context of cooperative games with transferable utility. Cooperative games are widely applied in economic allocation problems such as auction games (see Graham et al. 1990), airport landing fee problems (see Littlechild and Owen 1973), water distribution problems (see Ambec and Sprumont 2002), polluted river problems (see Ni and Wang 2007), sequencing problems (see Curiel et al. 1989) and queueing problems (see Maniquet 2003). For these games the trade-off between marginalism and egalitarianism can be seen as the trade-off between allocating according to the Shapley value or the equal division solution. Both solutions consider situations where eventually the 'grand coalition' consisting of all players forms. The Shapley value (Shapley 1953) assigns to every player its expected marginal contribution assuming that all possible orders of entrance of the players occur with equal probability. Here, the marginal contribution of a player to a coalition is the increase in transferable utility when this player joins the coalition. The marginalist characteristic of the Shapley value is most clearly formulated by Young (1985) who characterized it as the unique solution that is efficient, symmetric and strongly monotonic. This strong monotonicity states that the payoffs of a player do not decrease if its marginal contributions to coalitions do not decrease. Since symmetry (i.e. equal treatment of equals) is usually considered a desirable property, and under complete information also efficiency is widely accepted, this result says that the Shapley value is the only solution in which the utility payoff of a player is fully determined by its marginal contributions to the transferable utility of coalitions. ${ }^{1}$

On the other hand, the equal division solution which allocates the worth of the grand coalition equally among all players, can be seen as the most egalitarian solution for such games. The trade-off between marginalism and egalitarianism can be made by considering convex combinations of the Shapley value and the equal division solution. This class of solutions is introduced by Joosten (1996) and are called egalitarian Shapley values. Surprisingly, it turns out that these solutions can be characterized by similar axioms and implementations. We provide three types of results that are very common, yet distinctive, in game theory: consistency, monotonicity and implementation. ${ }^{2}$

First, we show that all these solutions satisfy the reduced game consistency that is used by Sobolev (1973) to characterize the Shapley value. This result is quite intriguing as these seemingly contrastive solutions have this well-known consistency in common. In these reduced games, after a particular player leaves the game with its payoff, the remaining coalitions assume with a probability that is proportional to their cardinality that the leaving player cooperates with them or not. Reduced game consistency requires that players respect the recommendations made by the solution in the sense that the solution assigns to the players in the reduced game the same payoffs as it assigns to those players in the original game. Sobolev (1973) characterized the Shapley value as

\footnotetext{
1 As already noticed by Young (1985) it is sufficient to weaken strong monotonicity to marginalism stating that the payoff of a player in two games is equal if all its marginal contributions are equal in both games.

2 Another 'tension' in cooperative games is the one between egalitarianism and participation constraints as studied in Dutta and Ray (1989).
} 
the unique solution that satisfies consistency and standardness for two-player games, the latter axiom meaning that in a two-player game every player earns its own worth plus half of what remains of the worth of the two-player ('grand') coalition. An inspiring result we found is that all egalitarian Shapley values satisfy Sobolev's consistency. Since the Shapley value is the unique egalitarian Shapley value satisfying standardness for two-player games, one can deduce that the connection as well as difference among all egalitarian Shapley values might lie in an alternative standardness for two-player games. An intuitive way to consider such a standardness is to assign to both players a uniform fraction $\alpha$ in their singleton worth and distribute the remainder equally among them. It turns out that for any $\alpha \in[0,1]$ the corresponding egalitarian Shapley value is characterized by consistency and the corresponding standardness axiom. Since all egalitarian Shapley values share the same reduced game consistency, the difference thus boils down to the allocation that is applied in two-player games.

The second characterization is based on monotonicity properties. Above we already referred to the characterization of the Shapley value by efficiency, symmetry and strong monotonicity (or marginalism) in Young (1985). Since all egalitarian Shapley values satisfy efficiency and symmetry, this implies that the Shapley value is the unique egalitarian Shapley value that is strongly monotonic. However, it turns out that all egalitarian Shapley values satisfy the weaker property which states that the payoff of a player does not decrease if its marginal contributions do not decrease and, moreover, the worth of the 'grand coalition' does not decrease. This is a considerable weakening of the strong monotonicity property. Since the worth of the 'grand coalition' is what is to be allocated by any efficient solution, strong monotonicity requires that the payoff of a player does not decrease if its marginal contributions do not decrease irrespective of what is to be allocated, which is a very strong requirement. However, if the worth to be allocated is not decreasing then this requirement on the payoffs seems reasonable for a marginalistic solution. We show that the class of egalitarian Shapley values is characterized by this weak monotonicity together with the well-known properties of efficiency, anonymity and linearity. Although these axioms are logically independent, we also provide an axiomatization without linearity. Instead of linearity we then use a weak covariance property stating that only adding a constant to every coalition that contains a particular player, has the same effect on the payoffs of all other players. This shows the important result that, although the Shapley value is the only solution in this class that satisfies strong monotonicity (which expresses marginalism), the egalitarian Shapley values are characterized using a weaker monotonicity property where besides the marginal contributions also the worth of the 'grand coalition' is taken into account.

Whereas the above two characterizations of the egalitarian Shapley values give a cooperative foundation (on a variable, respectively, fixed player set), our third result provides a non-cooperative foundation by implementing the egalitarian Shapley values as the unique subgame perfect equilibrium outcome in an extensive form bidding mechanism. This bidding mechanism generalizes the one for the Shapley value given in Pérez-Castrillo and Wettstein (2001), differing only in an additional possibility of breakdown of the negotiations. The implementation result verifies the intuition that the negotiation outcome tends to be more egalitarian if it has a higher probability of breakdown. 
Key differences and connections between fundamental concepts and theories may lie in the underlying details of the relevant notions. In a recent paper, Salles (2008) develops our understanding of rights and freedom. By replacing liberalism with weak liberalism, a constructive result is obtained in the context of social decision functions, contrasting to the well-known Sen's impossibility theorem (1970). In a similar spirit, our paper presents the above three results to discover the major differences between marginalism and egalitarianism and also to establish the long neglected close relationships between them although they look rather far from each other.

The paper is organized as follows. Section 2 discusses some preliminaries on cooperative games with transferable utility and solutions, including the egalitarian Shapley values. In Sect. 3 we provide the characterization using standardness and reduced game consistency. In Sect. 4 we provide a characterization of the class of egalitarian Shapley values using monotonicity properties. In Sect. 5 we provide an implementation of the egalitarian Shapley values. Finally, Sect. 6 contains some concluding remarks.

\section{Preliminaries}

A situation in which a finite set of players can obtain certain payoffs by cooperation can be described by a cooperative game with transferable utility, or simply a TU-game, being a pair $(N, v)$, where $N \subset \mathbb{N}$ is a finite set of players and $v: 2^{N} \rightarrow \mathbb{R}$ is a characteristic function on $N$ such that $v(\emptyset)=0$. For any coalition $S \subseteq N, v(S)$ is called the worth of coalition $S$. This is the transferable utility that the members of coalition $S$ can obtain by agreeing to cooperate. We denote the class of all TU-games by $\mathcal{G}$. A TU-game $(N, v)$ is monotone if $v(S) \leq v(T)$ whenever $S \subseteq T \subseteq N$. The unanimity game of coalition $T \subseteq N, T \neq \emptyset$, on $N$ is the monotone game $\left(N, u_{T}\right)$ given by $u_{T}(S)=1$ if $T \subseteq S$, and $u_{T}(S)=0$ otherwise. In the sequel we denote $n=|N|$ for the number of players in $N$. For generic coalitions $S \subseteq N$ we denote $s=|S|$.

A payoff vector of game $(N, v)$ is an $n$-dimensional real vector $x \in \mathbb{R}^{n}$ which represents a distribution of the payoffs that can be earned by cooperation over the individual players. A (point-valued) solution for TU-games is a function $\psi$ which assigns a payoff vector $\psi(N, v) \in \mathbb{R}^{n}$ to every TU-game $(N, v) \in \mathcal{G}$ such that $\psi_{i}(\{i\}, v)=v(\{i\})$ for all $i \in \mathbb{N}$. Two well-known solutions are the Shapley value and the equal division solution. The Shapley value (Shapley 1953) is the solution that assigns to every player $i \in N$ in TU-game $(N, v)$ its expected marginal contribution assuming that all possible orders of entrance of the players to the 'grand coalition' occur with equal probability,

$$
S h_{i}(N, v)=\sum_{\substack{S \subseteq N \\ i \in S}} \frac{(n-s) !(s-1) !}{n !}(v(S)-v(S \backslash\{i\})) \quad \text { for all } i \in N .
$$

The equal division solution is the solution that distributes the worth $v(N)$ of the 'grand coalition' equally among all players and thus assigns to every TU-game $(N, v)$ the payoff vector 


$$
E D_{i}(N, v)=\frac{v(N)}{n} \text { for all } i \in N
$$

Joosten (1996) introduced a new class of solutions that are obtained as convex combinations of the Shapley value and the equal division solution. For every $\alpha \in[0,1]$, he defines the $\alpha$-egalitarian Shapley value $\varphi^{\alpha}$ as the solution given by

$$
\varphi^{\alpha}(N, v)=\alpha \operatorname{Sh}(N, v)+(1-\alpha) E D(N, v) .
$$

We refer to a generic $\alpha$-egalitarian Shapley value as an egalitarian Shapley value. Some well-known properties of solutions for TU-games are the following. Solution $\psi$

- $\quad$ is efficient ${ }^{3}$ if $\sum_{i \in N} \psi_{i}(N, v)=v(N)$ for all $(N, v) \in \mathcal{G}$;

- $\quad$ is linear if $\psi(N, a v+b w)=a \psi(N, v)+b \psi(N, w)$ for all $(N, v),(N, w) \in \mathcal{G}$, where $a v+b w$ is given by $(a v+b w)(S)=a v(S)+b w(S)$ for all $S \subseteq N$;

- $\quad$ is symmetric if $\psi_{i}(N, v)=\psi_{j}(N, v)$ for all $(N, v) \in \mathcal{G}$ and $i, j \in N$ such that $v(S \cup\{i\})=v(S \cup\{j\})$ for all $S \subseteq N \backslash\{i, j\}$;

- $\quad$ satisfies anonymity if $\psi_{i}(N, v)=\psi_{\pi(i)}(N, \pi v)$ for all games $(N, v) \in \mathcal{G}$ and all permutations $\pi$ on $N$, where game $(N, \pi v)$ is defined by $\pi v\left(\bigcup_{i \in S}\{\pi(i)\}\right)=v(S)$ for all $S \subseteq N$;

- $\quad$ satisfies strong monotonicity if $\psi_{i}(N, v) \geq \psi_{i}(N, w)$ for every pair of games $(N, v),(N, w)$ and $i \in N$ such that $v(S \cup\{i\})-v(S) \geq w(S \cup\{i\})-w(S)$ for all $S \subseteq N \backslash\{i\}$

- $\quad$ satisfies $\alpha$-standardness for two-player games, $\alpha \in[0,1]$, if for every $(N, v) \in \mathcal{G}$ with $N=\{i, j\}, i \neq j$, it holds that $\psi_{i}(N, v)=\alpha v(\{i\})+\frac{1}{2}(v(N)-\alpha v(\{i\})-$ $\alpha v(\{j\}))=\frac{\alpha}{2}(v(\{i\})-v(\{j\}))+\frac{1}{2} v(N)$;

- $\quad$ is covariant if $\psi_{i}(N, w)=a \psi_{i}(N, v)+b$ for every $(N, v) \in \mathcal{G}, a \in \mathbb{R}$ and $b \in \mathbb{R}^{n}$, where $w$ is given by $w(S)=a v(S)+\sum_{j \in S} b_{j}$ for all $S \subseteq N$.

Specific choices of $\alpha \in[0,1]$ give different versions of standardness for twoplayer games as encountered in the literature. Taking $\alpha=1$ yields standardness for two-player games as considered in, e.g. Hart and Mas-Colell $(1988,1989): \psi_{i}(N, v)=$ $\frac{1}{2} v(\{i\})-\frac{1}{2} v(\{j\})+\frac{1}{2} v(N)=v(\{i\})+\frac{1}{2}(v(N)-v(\{i\})-v(\{j\}))$ with $N=\{i, j\}$. Taking $\alpha=0$, yields egalitarian standardness for two-player games: $\psi_{i}(N, v)=\frac{1}{2} v(N)$ for $i \in N$.

It is known that the Shapley value satisfies standardness and the equal division solution satisfies egalitarian standardness for two-player games. Joosten (1996) showed that for $\alpha \in[0,1]$, the $\alpha$-egalitarian Shapley value $\varphi^{\alpha}$ satisfies the corresponding $\alpha$-standardness for two-player games.

\section{Consistency}

In the literature various reduced game properties are discussed. Suppose that one player leaves a game with some payoff. Reduced games (in relation to the original game) describe what games are played by the remaining players, i.e. what is earned by

\footnotetext{
${ }_{3}$ Efficient solutions are often called values.
} 
coalitions of the remaining players after one player has left the game. The worths of coalitions in the reduced games usually depend on (i) the worths that these coalitions could earn on their own in the original game, (ii) what these coalitions could earn with the leaving player and (iii) the payoff with which the leaving player left the game. One of the oldest reduced games is the following that is introduced by Sobolev (1973).

Definition 3.1 Given game $(N, v) \in \mathcal{G}$, player $j \in N$, and efficient payoff vector $x \in \mathbb{R}^{n}$, the reduced game with respect to $j$ and $x$ is the game $\left(N \backslash\{j\}, v^{x}\right)$ given by

$$
v^{x}(S)=\frac{s}{n-1}\left(v(S \cup\{j\})-x_{j}\right)+\frac{n-1-s}{n-1} v(S) \text { for all } S \subseteq N \backslash\{j\} .
$$

An intuitive interpretation is as follows. Consider a game $(N, v)$, a payoff vector $x \in \mathbb{R}^{n}$, and a player $j \in N$. Suppose player $j$ leaves the game. Then, all remaining players will play a reduced game in relation to the original game, where all subcoalitions of $N \backslash\{j\}$ need to consider the possible payoffs for them after paying $j$ according to $x$. To compute the worth of a coalition $S \subseteq N \backslash\{j\}$ in the reduced game, according to Sobolev (1973), it is assumed that $S$ has two possibilities: it (i) either has the cooperation of the leaving player $j$, but must pay for $j$ 's payoff and thus earns $v(S \cup\{j\})-x_{j}$, or (ii) is on its own and earns its own worth $v(S)$. The reason why $j$ is still considered to have cooperation with a coalition $S$ is due to the idea of the reduced game itself. Since the reduced game is obtained from the original game by taking into account the payoff $x_{j}$ offered to $j$, we need to identify where $x_{j}$ comes from and who should pay for it. Generally, the payoff $x_{j}$ comes from a cooperation between $j$ and a coalition of the other players, rather than $j$ herself as otherwise she would simply be offered $v(\{j\})$. Consequently, every coalition $S \subseteq N \backslash\{j\}$ in the reduced game assumes that it cooperates with $j$ with a positive probability which depends on the cardinality of $S$. That is, $\frac{s}{n-1}$ represents the probability that coalition $S$ can have the cooperation of $j$ and thus earns $v(S \cup\{j\})-x_{j}$, and with probability $1-\frac{s}{n-1}=\frac{n-1-s}{n-1}$ coalition $S$ is on its own and earns $v(S)$. Naturally, if $S=N \backslash\{j\}$, which implies that all remaining players of $N \backslash\{j\}$ jointly agree to work with $j$, then for sure the cooperation is realized and $x_{j}$ is paid by $N \backslash\{j\}$, i.e. $v^{x}(N \backslash\{j\})=v(N)-x_{j}$.

Consistency with respect to a particular reduced game means that given a game $(N, v)$, if $x$ is a solution payoff vector for $(N, v)$, then for every player $j \in N$, the payoff vector $x_{N \backslash\{j\}}$ with payoffs for the players in $N \backslash\{j\}$, must be a solution payoff vector of the reduced game $\left(N \backslash\{j\}, v^{x}\right)$. It is a kind of internal consistency requirement to guarantee that players respect the recommendations made by the solution. In the following we refer to consistency with respect to the reduced game defined in Definition 3.1 just as consistency. ${ }^{4}$

Definition 3.2 Let $\psi$ be a solution on $\mathcal{G}$. Solution $\psi$ satisfies consistency on $\mathcal{G}$ if and only if for every $(N, v) \in \mathcal{G}$ with $n \geq 2, j \in N$, and $x=\psi(N, v)$, it holds that $\psi_{i}\left(N \backslash\{j\}, v^{x}\right)=\psi_{i}(N, v)$ for all $i \in N \backslash\{j\}$.

\footnotetext{
${ }^{4}$ We only consider the class $\mathcal{G}$ of all TU-games. If one considers subclasses $\mathcal{C} \subset \mathcal{G}$, then in the definition of consistency one should additionally require that the reduced games $\left(N \backslash\{j\}, v^{x}\right)$ also belong to $\mathcal{C}$.
} 
Sobolev (1973) showed that the Shapley value is the unique solution that satisfies this consistency and is standard for two-player games. Surprisingly, also the equal division solution satisfies this consistency. It turns out that this even holds more general for all egalitarian Shapley values. To facilitate the proof of this result, we like to note that one can readily verify that an $\alpha$-egalitarian Shapley value of a game $v$ can be expressed as the Shapley value of a modified game that is the corresponding convex combination of the original game $v$ and the game $f(v)$, where $f(v)(S)=v(N)$ if $S=N$, and $f(v)(S)=0$ for all $S \subset N$. Note that $f(v)$ is actually $v(N)$ times the unanimity game of $N$, i.e., $f(v)=v(N) u_{N}$. That is, for every TU game $(N, v)$ and any $\alpha \in[0,1]$, the egalitarian Shapley value $\varphi^{\alpha}(N, v)=\operatorname{Sh}(N, \alpha v+(1-\alpha) f(v))^{5}$

Proposition 3.3 Every egalitarian Shapley value $\varphi^{\alpha}, \alpha \in[0,1]$, satisfies consistency.

Proof Take $j \in N, S \subseteq N \backslash\{j\}$ and let $x=\varphi^{\alpha}(N, v), y=\operatorname{Sh}(N, v)$, and $z=$ $S h(N, f(v))$. (Note that $z$ also equals $E D(N, f(v))$. Recall that $n=|N|$ and $s=|S|$. We have that, for every $i \in N$,

$$
\begin{aligned}
\varphi_{i}^{\alpha}\left(N \backslash\{j\}, v^{x}\right)= & S h_{i}\left(N \backslash\{j\}, \alpha v^{x}+(1-\alpha) f\left(v^{x}\right)\right) \\
= & \alpha S_{i}\left(N \backslash\{j\}, v^{x}\right)+(1-\alpha) S h_{i}\left(N \backslash\{j\}, f\left(v^{x}\right)\right) \\
= & \alpha S h_{i}\left(N \backslash\{j\}, \alpha v^{y}+(1-\alpha) v^{z}\right)+(1-\alpha) \frac{v(N)-\varphi_{j}^{\alpha}(N, v)}{n-1} \\
= & \alpha S_{i}\left(N \backslash\{j\}, v^{y}\right)+\alpha(1-\alpha) S h_{i}\left(N \backslash\{j\}, v^{z}-v^{y}\right) \\
& +(1-\alpha) \frac{v(N)-\frac{v(N)}{n}+\alpha\left(\frac{v(N)}{n}-S_{j}(N, v)\right)}{n-1} \\
= & \alpha \operatorname{Sh}_{i}\left(N \backslash\{j\}, v^{y}\right)+(1-\alpha) \frac{v(N)-\frac{v(N)}{n}}{n-1} \\
= & \alpha \operatorname{Sh}_{i}(N, v)+(1-\alpha) \operatorname{Sh}_{i}(N, f(v)) \\
= & S_{i}(N, \alpha v+(1-\alpha) f(v))=\varphi_{i}^{\alpha}(N, v) .
\end{aligned}
$$

Here, the first and last equalities follow from the definitions of egalitarian Shapley value and $f$. The second, fourth and seventh equalities follow from linearity of the Shapley value. The third equality follows from the definitions of the reduced games with respect to $x, y$ and $z$. For the fifth equality, please notice that $S h_{i}\left(N \backslash\{j\}, v^{z}-\right.$ $\left.v^{y}\right)=\frac{S h_{j}(N, v)-\frac{v(N)}{n}}{n-1}$ because by definition of the reduced games, the game $(N \backslash$ $\left.\{j\}, v^{z}-v^{y}\right)$ is defined by $\left(v^{z}-v^{y}\right)(N \backslash\{j\})=S h_{j}(N, v)-\frac{v(N)}{n}$ and $\left(v^{z}-v^{y}\right)(S)=$ $\frac{s}{n-1}\left(S h_{j}(N, v)-\frac{v(N)}{n}\right)$ for all $S \subset N \backslash\{j\}$. Since all players in $N \backslash\{j\}$ are symmetric in this game, we know that $S h_{i}\left(N \backslash\{j\}, v^{z}-v^{y}\right)=\frac{S h_{j}(N, v)-\frac{v(N)}{n}}{n-1}$. The sixth equality follows from the fact that the Shapley value is consistent and by the definition of $f$.

\footnotetext{
5 We are grateful to the referee for this valuable suggestion that helps a lot in simplifying the corresponding proofs in the paper and making the results more transparent.
} 
Sobolev (1973) characterized the Shapley value as the unique solution that satisfies consistency and standardness for two-player games. Since in Proposition 3.3 we showed that all egalitarian Shapley values satisfy the same consistency, the Shapley value is the unique egalitarian Shapley value satisfying standardness for two-player games. Therefore, we need to adapt standardness for two-player games to obtain a characterization for other (Sobolev) consistent solutions than the Shapley value. An intuitive way is to assign to both players a uniform fraction in their singleton worth and distribute the remainder equally among them, as expressed by $\alpha$-standardness for two-player games in the previous section. It turns out that every $\alpha$-egalitarian Shapley value satisfies the corresponding $\alpha$-standardness for two-player games. For any $\alpha \in[0,1]$ these two axioms characterize the corresponding egalitarian Shapley value. $^{6}$

Theorem 3.4 Take any $\alpha \in[0,1]$. A solution $\psi$ satisfies consistency and $\alpha$-standardness for two-player games if and only if $\psi=\varphi^{\alpha}$.

Proof Since it is straightforward that $\varphi^{\alpha}$ satisfies $\alpha$-standardness for two-player games, by Proposition 3.3 we are left to show uniqueness. Suppose that solution $\psi$ satisfies the two properties of the theorem. We will show that $\psi(N, v)=\varphi^{\alpha}(N, v)$ for any game $(N, v) \in \mathcal{G}$. For $n=1$, by definition of a solution we have $\psi_{i}(\{i\}, v)=v(\{i\})$ for all $i \in \mathbb{N}$. For $n=2, \alpha$-standardness of $\psi$ and $\varphi^{\alpha}$ implies that they are equal. Proceeding by induction, suppose that $\psi\left(N^{\prime}, v^{\prime}\right)=\varphi^{\alpha}\left(N^{\prime}, v^{\prime}\right)$ whenever $2 \leq\left|N^{\prime}\right|<n$.

Let $x=\psi(N, v)$ and $y=\varphi^{\alpha}(N, v)$. Take any $i, j \in N, i \neq j$, and consider the two reduced games $\left(N \backslash\{j\}, v^{x}\right),\left(N \backslash\{j\}, v^{y}\right)$. Then,

$$
\begin{aligned}
x_{i}-y_{i} & =\psi_{i}(N, v)-\varphi_{i}^{\alpha}(N, v) \\
& =\psi_{i}\left(N \backslash\{j\}, v^{x}\right)-\varphi_{i}^{\alpha}\left(N \backslash\{j\}, v^{y}\right) \\
& =\varphi_{i}^{\alpha}\left(N \backslash\{j\}, v^{x}\right)-\varphi_{i}^{\alpha}\left(N \backslash\{j\}, v^{y}\right) \\
& =\alpha\left(S_{i}\left(N \backslash\{j\}, v^{x}-v^{y}\right)\right)+(1-\alpha)\left(S h_{i}\left(N \backslash\{j\}, f\left(v^{x}\right)-f\left(v^{y}\right)\right)\right) \\
& =\alpha \frac{y_{j}-x_{j}}{n-1}+(1-\alpha) \frac{y_{j}-x_{j}}{n-1}=\frac{y_{j}-x_{j}}{n-1},
\end{aligned}
$$

where the second equality follows from the fact that both solutions $\psi$ and $\varphi^{\alpha}$ satisfy consistency, the third equality follows by the induction hypothesis, and the fourth equality follows by the definition of $\varphi^{\alpha}$ as well as linearity of the Shapley value. For the fifth equality, one can readily verify that the game $\left(N \backslash\{j\}, v^{x}-v^{y}\right)$ is defined by $\left(v^{x}-v^{y}\right)(N \backslash\{j\})=y_{j}-x_{j}$ and $\left(v^{z}-v^{y}\right)(S)=\frac{s}{n-1}\left(y_{j}-x_{j}\right)$ for all $S \subset N \backslash\{j\}$. Obviously, all players are symmetric in this game. Thus, $S h_{i}\left(N \backslash\{j\}, v^{x}-v^{y}\right)=\frac{y_{j}-x_{j}}{n-1}$.

Since this holds for all $i, j \in N, i \neq j$, and $n \geq 3$, it necessarily leads to that $x_{i}-y_{i}=0$ for all $i \in N$, and thus $\psi(N, v)=\varphi^{\alpha}(N, v)$.

\footnotetext{
${ }^{6}$ Uniqueness of a solution satisfying $\alpha$-standardness for two-player games and consistency also follows from Yanovskaya and Driessen (2002). We give an explicit proof of uniqueness since this is much shorter with the explicit reduced game of Sobolev, and gives insight in the particular class of solutions we consider here.
} 
In van den Brink and Funaki (2009) it is shown that for two-player games, a solution satisfies $\alpha$-standardness for some $\alpha \in[0,1]$ if and only if it is efficient, symmetric and linear. Hence, by the above theorem the class of $\alpha$-egalitarian Shapley values is characterized by consistency, efficiency, symmetry and linearity.

It is important to notice that the consistency condition is not linear for linear and consistent solutions, meaning that a linear combination of two linear consistent solutions need not be consistent. To show this, consider two solutions, $\psi$ and $\varphi$, which satisfy linearity and consistency, and a game $(N, v) \in \mathcal{G}$. Putting $x=\psi(N, v)$ and $y=\varphi(N, v)$, then for $j \in N$ and $\alpha \in[0,1]$,

$$
\begin{aligned}
(\alpha \psi+(1-\alpha) \varphi)(N, v) & =\alpha \psi(N, v)+(1-\alpha) \varphi(N, v) \\
& =\alpha \psi\left(N \backslash\{j\}, v^{x}\right)+(1-\alpha) \varphi\left(N \backslash\{j\}, v^{y}\right) .
\end{aligned}
$$

By linearity of $(\alpha \psi+(1-\alpha) \varphi)$, we have $(\alpha \psi+(1-\alpha) \varphi)(N, v)=\alpha x+(1-\alpha) y$, and thus

$$
\begin{aligned}
(\alpha \psi+(1-\alpha) \varphi)\left(N \backslash\{j\}, v^{\alpha x+(1-\alpha) y}\right)= & \alpha \psi\left(N \backslash\{j\}, v^{\alpha x+(1-\alpha) y}\right) \\
& +(1-\alpha) \varphi\left(N \backslash\{j\}, v^{\alpha x+(1-\alpha) y}\right) .
\end{aligned}
$$

Since, in general, the right hand sides of Eqs. 3.1 and 3.2 are different $^{7}$, the solution $(\alpha \psi+(1-\alpha) \varphi)$, in general, does not satisfy consistency. This makes the characterization of the egalitarian Shapley values stated in Theorem 3.4 even more surprising.

The reduced game of Sobolev (1973) is not the only reduced game with respect to which the Shapley value is consistent. For example, the Shapley value satisfies consistency with respect to the reduced game of Hart and Mas-Colell $(1988,1989) .{ }^{8}$ However, the Shapley value is the only egalitarian Shapley value that is Hart and MasColell consistent. Joosten (1996) characterized the $\alpha$-egalitarian Shapley value using $\alpha$-standardness for two-player games and an adapted version of Hart and Mas-Colell's reduced game consistency where the reduced game depends on the parameter $\alpha$. The main disadvantage of that characterization therefore is that the parameter $\alpha$ appears both in the standardness and in the reduced game consistency property. However, we have shown here that all $\alpha$-egalitarian Shapley values have the same reduced game consistency property in common when considering Sobolev (1973)'s reduced game.

Theorem 3.4 helps to advance our insight on the fundamental economic and social concepts of marginalism and egalitarianism. Both seemingly completely opposite extremes possess the same consistency. The only difference is how we treat the basic unit of a society, i.e., a situation containing two players only.

\footnotetext{
7 This is even the case, when $v^{\alpha x+(1-\alpha) y}=\alpha v^{x}+(1-\alpha) v^{y}$ (which is satisfied by our reduced game), since the right hand side of Eq. 3.2 becomes $\alpha^{2} \psi\left(N \backslash\{j\}, v^{x}\right)+\alpha(1-\alpha) \psi\left(N \backslash\{j\}, v^{y}\right)+(1-\alpha) \alpha \varphi(N \backslash$ $\left.\{j\}, v^{x}\right)+(1-\alpha)^{2} \varphi\left(N \backslash\{j\}, v^{y}\right)$.

${ }^{8}$ In this reduced game it is also possible for coalitions of more than one player to leave the game. For a given game $(N, v) \in \mathcal{G}$ with $n \geq 3$, a coalition $T \subseteq N$, and an efficient payoff vector $x \in \mathbb{R}^{n}$, the Hart and Mas-Colell reduced game $\left(T, v^{x}\right)$ is given by $v^{x}(S)=v\left(S \cup T^{c}\right)-\sum_{j \in T^{c}} x_{T^{c}}$ for all $S \subseteq T, S \neq \emptyset$, where $T^{c}=N \backslash T$.
} 


\section{Monotonicity}

Several monotonicity properties of solutions have been discussed in the literature. For example, Young (1985) showed that the Shapley value is characterized by efficiency, symmetry and strong monotonicity. Since all egalitarian Shapley values are efficient and symmetric, the Shapley value thus is the only egalitarian Shapley value that satisfies strong monotonicity. On the other hand, van den Brink (2007) characterized the equal division solution by efficiency, symmetry and coalitional monotonicity ${ }^{9}$, and thus the equal division solution is the only egalitarian Shapley value that satisfies coalitional monotonicity.

Since the worth of the 'grand coalition' is what is to be allocated by any efficient solution, requiring that the payoff of a player does not decrease if its marginal contributions do not decrease (as required by strong monotonicity) irrespective of what is to be allocated is a very strong requirement. For example, the payoff of a player does not decrease if its marginal contributions do not decrease even when instead of allocation a surplus of 100 , the players together have to pay a cost of 100 . Usually, in allocation problems also the worth of the 'grand coalition' has an effect on the payoff distribution. However, if we change a game such that not only the marginal contributions of a player, but also the worth of the 'grand coalition' does not decrease, then requiring that the payoff of that player does not decrease seems reasonable.

Axiom 4.1 (Weak monotonicity) A solution $\psi$ satisfies weak monotonicity if $\psi_{i}(N, v) \geq \psi_{i}(N, w)$ whenever $v(N) \geq w(N)$ and $v(S)-v(S \backslash\{i\}) \geq w(S)-$ $w(S \backslash\{i\})$ for all $S \subseteq N$ with $i \in S$.

It turns out that all egalitarian Shapley values satisfy this weaker monotonicity property. Moreover, the class of egalitarian Shapley values can be characterized using this property. To prove this we first show that weak monotonicity and anonymity imply local monotonicity, meaning that whenever the marginal contributions of a player $i$ are greater or equal to those of player $j$ in a game, then the payoff of player $i$ is at least equal to the payoff of player $j$.

Lemma 4.2 If a solution $\psi$ satisfies weak monotonicity and anonymity, then $\psi_{i}(N, v) \geq \psi_{j}(N, v)$ for all $(N, v) \in \mathcal{G}$ and $i, j \in N$ such that $v(S \cup\{i\}) \geq v(S \cup\{j\})$ for all $S \subseteq N \backslash\{i, j\}$.

Proof Let $\psi$ be a solution that satisfies weak monotonicity and anonymity. Take any game $(N, v) \in \mathcal{G}$, and suppose that $i$ and $j$ satisfy $v(S \cup\{i\}) \geq v(S \cup\{j\})$ for all $S \subseteq N \backslash\{i, j\}$

Consider a permutation $\pi$ of $N$ such that $\pi(i)=j, \pi(j)=i, \pi(k)=k$ for $k \in N \backslash\{i, j\}$, and denote $w(S)=\pi v(S)$ for all $S \subseteq N$. Then

- $w(S)=v(S)$ if $i, j \in S$ or $S \subseteq N \backslash\{i, j\}$,

- $w(S)=v((S \cup\{i\}) \backslash\{j\})$ if $j \in S \subseteq N \backslash\{i\}$, and

- $w(S)=v((S \cup\{j\}) \backslash\{i\})$ if $i \in S \subseteq N \backslash\{j\}$.

\footnotetext{
9 Solution $\psi$ satisfies coalitional monotonicity if $\psi_{i}(N, v) \geq \psi_{i}(N, w)$ for every pair of games $(N, v),(N, w)$ and $i \in N$ such that $v(S) \geq w(S)$ for all $S \subseteq N$ with $i \in S$.
} 
Take any $S \subseteq N$ such that $i \in S$. If $j \in S$, then we have

$$
\begin{aligned}
& (v(S)-v(S \backslash\{i\}))-(w(S)-w(S \backslash\{i\})) \\
& \quad=(v(S)-v(S \backslash\{i\}))-(v(S)-v(S \backslash\{j\}))=v(S \backslash\{j\})-v(S \backslash\{i\}) \geq 0 .
\end{aligned}
$$

If $S \subseteq N \backslash\{j\}$, then we have

$$
\begin{aligned}
& (v(S)-v(S \backslash\{i\}))-(w(S)-w(S \backslash\{i\})) \\
& =(v(S)-v(S \backslash\{i\}))-(v((S \cup\{j\}) \backslash\{i\})-v(S \backslash\{i\})) \\
& =v(S)-v((S \cup\{j\}) \backslash\{i\}) \geq 0 .
\end{aligned}
$$

Since $v(N)=w(N)$, weak monotonicity implies that $\psi_{i}(N, v) \geq \psi_{i}(N, w)$. By anonymity we have $\psi_{i}(N, w)=\psi_{i}(N, \pi v)=\psi_{j}(N, v)$, and thus $\psi_{i}(N, v) \geq \psi_{j}(N, v)$. This shows that $\psi$ satisfies local monotonicity.

Next we state our first main result of this section, characterizing the class of egalitarian Shapley values.

Theorem 4.3 A solution $\psi$ satisfies efficiency, linearity, anonymity and weak monotonicity if and only if it is an egalitarian Shapley value.

Proof It is straightforward to verify that all egalitarian Shapley values satisfy these four properties. Now, suppose that solution $\psi$ satisfies these properties.

For one-player games $(\{i\}, v)$ the payoff $\psi_{i}(\{i\}, v)=v(\{i\})$ follows by definition of a solution.

If $(N, v)$ is a null game given by $v(S)=0$ for all $S \subseteq N$, then efficiency and anonymity imply that $\psi_{i}(N, v)=0$ for all $i \in N$.

Next, we consider unanimity games $\left(N, u_{T}\right) \in \mathcal{G}, T \subseteq N, T \neq \emptyset$. We prove uniqueness for unanimity games $\left(N, u_{T}\right)$ by induction on $|T|$. First, suppose that $|T|=1$. Anonymity implies that there is a $c^{*} \in \mathbb{R}$ such that $\psi_{j}\left(N, u_{T}\right)=c^{*}$ for all $j \in N \backslash T$. For $i \in T$, Lemma 4.2 implies that $\psi_{i}\left(N, u_{T}\right) \geq c^{*}$. So, for $i \in T$ we can write $\psi_{i}\left(N, u_{T}\right)=c^{*}+\alpha$ for some $\alpha \geq 0$. For $i \in T$, efficiency then implies that $\psi_{i}\left(N, u_{T}\right)=1-(n-1) c^{*}$, and thus $\alpha=1-(n-1) c^{*}-c^{*}=1-n c^{*}$. We obtain $c^{*}=\frac{1-\alpha}{n}$, and thus

$$
\psi_{i}\left(N, u_{T}\right)=\left\{\begin{aligned}
c^{*}+\alpha & =\frac{1-\alpha}{n}+\alpha & & \text { if } i \in T \\
c^{*} & =\frac{1-\alpha}{n} & & \text { if } i \in N \backslash T,
\end{aligned}\right.
$$

with $\alpha \geq 0$. Weak monotonicity and the fact that all players in a null game earn zero payoff, implies that $\psi_{j}\left(N, u_{T}\right) \geq 0$ for all $j \in N \backslash T$, and thus $\alpha \leq 1$. Since $E D_{i}\left(N, u_{T}\right)=\frac{1}{n}$ for all $i \in N, S_{i}\left(N, u_{T}\right)=1$ for $i \in T$, and $\operatorname{Sh}_{i}\left(N, u_{T}\right)=0$ for $i \in N \backslash T$, with (4.3) we have $\psi\left(N, u_{T}\right)=(1-\alpha) E D\left(N, u_{T}\right)+\alpha \operatorname{Sh}\left(N, u_{T}\right)=$ $\varphi^{\alpha}\left(N, u_{T}\right), \alpha \in[0,1]$.

By anonymity, it holds that there is an $\alpha \in[0,1]$ such that $\psi\left(N, u_{T}\right)=\varphi^{\alpha}\left(N, u_{T}\right)$ for every $T \subset N$ with $|T|=1$. 
Now, consider $\left(N, u_{T}\right), 2 \leq|T|<n$. Proceeding by induction assume that we determined $\psi\left(N, u_{T^{\prime}}\right)=\varphi^{\alpha}\left(N, u_{T^{\prime}}\right)$ for some $\alpha \in[0,1]$ whenever $\left|T^{\prime}\right|<|T|$. If $j \in N \backslash T$ then weak monotonicity implies that $\psi_{j}\left(N, u_{T}\right)=\psi_{j}\left(N, u_{T \backslash\{i\}}\right)$ for any $i \in T$. With the induction hypothesis it then follows that $\psi_{j}\left(N, u_{T}\right)=$ $\psi_{j}\left(N, u_{T \backslash\{i\}}\right)=\varphi_{j}^{\alpha}\left(N, u_{T \backslash\{i\}}\right)=\frac{1-\alpha}{n}=\varphi_{j}^{\alpha}\left(N, u_{T}\right)$. With efficiency it then follows that $\sum_{i \in T} \psi_{i}\left(N, u_{T}\right)=1-\sum_{j \in N \backslash T} \psi_{j}\left(N, u_{T}\right)=1-(n-|T|) \frac{1-\alpha}{n}$. By anonymity we then have that $\psi_{i}\left(N, u_{T}\right)=\frac{1-(n-|T|) \frac{1-\alpha}{n}}{|T|}=\frac{1-\alpha}{n}+\frac{\alpha}{|T|}=(1-\alpha) E D_{i}\left(N, u_{T}\right)+$ $\alpha \operatorname{Sh}_{i}\left(N, u_{T}\right)=\varphi_{i}^{\alpha}\left(N, u_{T}\right)$ for $i \in T$. Thus, $\psi\left(N, u_{T}\right)=\varphi^{\alpha}\left(N, u_{T}\right)$.

For the unanimity game $\left(N, u_{N}\right)$, anonymity and efficiency imply that $\psi_{i}(N, v)=$ $\frac{1}{n}$ for all $i \in N$.

For arbitrary $(N, v) \in \mathcal{G}$, uniqueness follows from linearity of $\psi$ and the fact that $v=\sum_{\substack{T \subseteq N \\ T \neq \emptyset}} \Delta_{v}(T) u_{T}$ for every $(N, v) \in \mathcal{G}$, with $\Delta_{v}(T)=\sum_{H \subseteq T}(-1)^{|T|-|H|} v(H)$ the Harsanyi dividend of coalition $T \subseteq N, T \neq \emptyset$ (see Harsanyi 1959).

Note that Theorem 4.3 characterizes the class of egalitarian Shapley values using axioms that are fully independent of the parameter $\alpha$. At first sight, it seems that requiring weak monotonicity and linearity is rather strong since Young (1985) characterized the Shapley value without linearity. However, we argued above that Young's strong monotonicity is considerably stronger than our weak monotonicity. In fact, the four axioms of Theorem 4.3 are logically independent, as shown by the following alternative solutions:

1. The solution $\psi$ given by $\psi_{i}(N, v)=0$ for all $i \in N$ and $(N, v) \in \mathcal{G}$ satisfies the axioms of Theorem 4.3 except efficiency.

2. Consider the solution $f$ given by $f(N, v)=\operatorname{Sh}(N, v)$ if $v(N) \leq 10$, and $f(N, v)=E D(N, v)$ if $v(N)>10$. This solution satisfies the axioms of Theorem 4.3 except linearity.

3. For $N \subset \mathbb{N}$, let $\bar{n}(N)$ be the lowest labeled player in $N$, i.e. $i \geq \bar{n}(N)$ for all $i \in N$. Then the solution $\psi$ given by $\psi_{\bar{n}(N)}(N, v)=v(N)-\sum_{i \in N \backslash\{\bar{n}(N)\}} v(\{i\})$, and $\psi_{i}(N, v)=v(\{i\})$ for all $i \in N \backslash\{\bar{n}(N)\}$ satisfies the axioms of Theorem 4.3 except anonymity.

4. The CIS-value given by $C I S_{i}(N, v)=v(\{i\})+\frac{v(N)-\sum_{j \in N} v(\{j\})}{n}$ for all $i \in N$ (see Driessen and Funaki 1991) satisfies the axioms of Theorem 4.3 except weak monotonicity.

Although the four axioms in Theorem 4.3 are logically independent, we want to search for an axiomatization of the egalitarian Shapley values using weak monotonicity but without linearity. It turns out that for games with at least three players it is possible by replacing linearity by a weak version of covariance. This weak covariance states that only adding a constant to every coalition that contains a particular player, has the same effect on the payoffs of all other players. (Note that covariance implies that this has no effect on the payoff of other players.)

Axiom 4.4 (Weak covariance) A solution $\psi$ satisfies weak covariance if there is a $c^{*} \in \mathbb{R}$ such that $\psi_{i}(N, v)-\psi_{i}(N, w)=c^{*}$ for all $i \in N \backslash\{j\}$ whenever for some $j \in N$ and $c \in \mathbb{R}, w$ is given by $w(S)=v(S)+c$ for all $S \subseteq N$ with $j \in S$, and $w(S)=v(S)$ for all $S \subseteq N \backslash\{j\}$. 
This axiom is also a weak version of fairness as used in van den Brink (2001) to characterize the Shapley value. ${ }^{10}$ Although the Shapley value is the only egalitarian Shapley value that satisfies covariance, all egalitarian Shapley values satisfy weak covariance. We denote by $\mathcal{G}_{3}$ the class of TU-games with at least three players.

Theorem 4.5 A solution $\psi$ satisfies efficiency, anonymity, weak monotonicity and weak covariance on $\mathcal{G}_{3}$ if and only if it is an egalitarian Shapley value.

Proof It is straightforward to verify that all egalitarian Shapley values satisfy weak covariance. Now, suppose that solution $\psi$ satisfies these properties on $\mathcal{G}_{3}$. Again, if $(N, v)$ is a null game given by $v(S)=0$ for all $S \subseteq N$, then efficiency and anonymity imply that $\psi_{i}(N, v)=0$ for all $i \in N$.

Next, we show uniqueness for inessential games, i.e. games $(N, v)$ such that $v(S)=$ $\sum_{i \in S} v(\{i\})$ for all $S \subseteq N$. For any inessential game $(N, v) \in \mathcal{G}_{3}$ define $H(N, v)=$ $\left\{j \in N \mid \Delta_{v}(\{j\}) \neq 0\right\}$ being the set of singleton coalitions with non-zero dividend, and $h(N, v)=|H(N, v)|$. We prove uniqueness by induction on $h(N, v)$. If $h(N, v)=1$ then $(N, v)$ is a unanimity game. Uniqueness of $\psi(N, v)$ follows similar as shown in the proof of Theorem 4.3. Proceeding by induction, assume that $\psi\left(N, v^{\prime}\right)$ is uniquely determined whenever $h\left(N, v^{\prime}\right)<h(N, v)$. Then there are least two players $i, j \in N$ with $\Delta_{v}(\{i\}) \neq 0$ and $\Delta_{v}(\{j\}) \neq 0$. Weak covariance implies that for every $g \in N \backslash\{i, j\}$ it holds that

$$
\psi_{i}(N, v)-\psi_{i}\left(N, v-\Delta_{v}(\{j\}) u_{\{j\}}\right)=\psi_{g}(N, v)-\psi_{g}\left(N, v-\Delta_{v}(\{j\}) u_{\{j\}}\right),
$$

and

$$
\psi_{j}(N, v)-\psi_{j}\left(N, v-\Delta_{v}(\{i\}) u_{\{i\}}\right)=\psi_{g}(N, v)-\psi_{g}\left(N, v-\Delta_{v}(\{i\}) u_{\{i\}}\right) .
$$

Since $h\left(N, v-\Delta_{v}(\{j\}) u_{\{j\}}\right)=h\left(N, v-\Delta_{v}(\{i\}) u_{\{i\}}\right)=h(N, v)-1$, the payoffs $\psi_{i}\left(N, v-\Delta_{v}(\{j\}) u_{\{j\}}\right), \psi_{g}\left(N, v-\Delta_{v}(\{j\}) u_{\{j\}}\right), \psi_{j}\left(N, v-\Delta_{v}(\{i\}) u_{\{i\}}\right)$ and $\psi_{g}\left(N, v-\Delta_{v}(\{i\}) u_{\{i\}}\right)$ are determined by the induction hypothesis. Taking all $(n-2)$ equations (4.4) (for every $g \in N \backslash\{i, j\}$ ), and taking one equation (4.5) (for some $g \in N \backslash\{i, j\})$, we have $(n-2)+1=n-1$ linearly independent equations. Together with efficiency, i.e.,

$$
\sum_{g \in N} \psi_{g}(N, v)=v(N)
$$

this yields $(n-1)+1=n$ linearly independent equations in the $n$ unknown payoffs, and thus $\psi(N, v)$ is uniquely determined.

Now, consider any game $(N, v) \in \mathcal{G}_{3}$. For any game $(N, v) \in \mathcal{G}_{3}$ define $\bar{H}(N, v)=$ $\left\{T \subset N \mid \Delta_{v}(T) \neq 0\right.$ and $\left.|T|>1\right\}$ being the set of coalitions with non-zero dividend and containing at least two players, and $\bar{h}(N, v)=|\bar{H}(N, v)|$. We prove uniqueness

$\overline{10}$ A solution $\psi$ satisfies fairness if $\psi_{i}(N, v+w)-\psi_{i}(N, v)=\psi_{j}(N, v+w)-\psi_{j}(N, v)$ whenever $i$ and $j$ are symmetric players in $(N, w)$. 
by induction on $\bar{h}(N, v)$. If $\bar{h}(N, v)=0$ then $(N, v)$ is an inessential game, and uniqueness of $\psi(N, v)$ is shown above.

Proceeding by induction, assume that $\psi\left(N, v^{\prime}\right)$ is uniquely determined whenever $\bar{h}\left(N, v^{\prime}\right)<\bar{h}(N, v)$. Let $D(N, v)=\{i \in N \mid S \in \bar{H}(N, v) \Rightarrow i \in S\}$ be the set of players that belong to every coalition that has at least two players and nonzero dividend. Take any $i \in N \backslash D(N, v)$. Then there is an $S \in \bar{H}(N, v)$ such that $i \notin S$. Take any $j \in N \backslash\{i\}$, and let $w=v+\Delta_{v}(S)\left(u_{\{j\}}-u_{S}\right)$. Note that $w(S)-w(S \backslash\{i\})=v(S)-v(S \backslash\{i\})$ for all $S \subseteq N$. Since also $w(N)=v(N)$, weak monotonicity implies that

$$
\psi_{i}(N, v)=\psi_{i}(N, w)=\psi_{i}\left(N, v+\Delta_{v}(S)\left(u_{\{j\}}-u_{S}\right)\right)
$$

Since $\bar{h}\left(N, v+\Delta_{v}(S)\left(u_{\{j\}}-u_{S}\right)\right)=\bar{h}(N, v)-1$, the payoff $\psi_{i}\left(N, v+\Delta_{v}(S)\left(u_{\{j\}}-\right.\right.$ $\left.u_{S}\right)$ ), and thus $\psi_{i}(N, v)$, is uniquely determined by the induction hypothesis.

Now, we are left to determine $\psi_{i}(N, v)$ for $i \in D(N, v)$. Let $m(v)=$ $\min _{j \in D(N, v)} v(\{j\})$, and define $M(N, v)=\{j \in D(N, v) \mid v(\{j\})=m(v)\}$. If $M(N, v)=D(N, v)$ then all players in $D(N, v)$ are symmetric, and thus anonymity implies that all $\psi_{i}(N, v), i \in D(N, v)$, are equal. Since we already determined the payoffs of players in $N \backslash D(N, v)$, efficiency determines $\psi(N, v)$.

Proceeding by a second induction, assume that $\psi\left(N, v^{\prime}\right)$ is determined whenever $\left|M\left(N, v^{\prime}\right)\right|>|M(N, v)|$. Take some $j \in D(N, v)$ with $v(\{j\})>m(v)$. Let $z=$ $v-(v(\{j\})-m(v)) u_{\{j\}}$.

Weak covariance implies that

$\psi_{g}(N, v)-\psi_{g}(N, z)=\psi_{i}(N, v)-\psi_{i}(N, z)$ for all $g \in D(N, v) \backslash\{j\}, i \in N \backslash D(N, v)$

(Note that $|D(N, v)| \geq 2$ since otherwise $|D(N, v)|=|M(N, v)|$.) Since $\psi_{i}(N, v)$ is determined above, and $\psi(N, z)$ is determined by the induction hypothesis, the payoff $\psi_{g}(N, v)$ is determined.

Since we already determined the payoffs $\psi_{i}(N, w)$ for all $i \in N \backslash D(N, v)$, efficiency determines $\psi_{j}(N, v)=v(N)-\sum_{g \in N \backslash\{j\}} \psi_{g}(N, v)$.

Logical independence of the four axioms of Theorem 4.5 can be shown by the same alternative solutions used to show logical independence of the axioms in Theorem 4.3.

Since the Shapley value is the only egalitarian Shapley value that satisfies covariance, strengthening weak covariance to covariance in Theorem 4.5 yields an axiomatization of the Shapley value using weak monotonicity.

Corollary 4.6 A solution $\psi$ satisfies efficiency, anonymity, weak monotonicity and covariance on $\mathcal{G}_{3}$ if and only if it is the Shapley value.

Theorem 4.5 characterizes the class of egalitarian Shapley values without linearity on the class of games with at least three players. Note that weak covariance has no meaning on the class of two-player games. To include also the two player games we might add the weak null player out property which is a weak consistency axiom stating that deleting a null player from a game changes the payoffs of all other players by the 
same amount. For $(N, v) \in \mathcal{G}$ and $T \subset N$, the restricted game $\left(T, v_{T}\right)$ is given by $v_{T}(S)=v(S)$ for all $S \subseteq T$.

Axiom 4.7 (Weak null player out property) A solution $\psi$ satisfies the weak null player out property if $\psi_{i}(N, v)-\psi_{i}\left(N \backslash\{h\}, v_{N \backslash\{h\}}\right)=\psi_{j}(N, v)-\psi_{j}\left(N \backslash\{h\}, v_{N \backslash\{h\}}\right)$ for every $i, j \in N$ and $h \in N \backslash\{i, j\}$ being a null player in $(N, v)$.

This is a weaker version of the null player out property as considered in Derks and Haller (1999) which states that deleting a null player does not change the payoffs of the other players. The weak version stated above is used in van den Brink and Funaki (2009) to characterize a class of equal division solutions.

Theorem 4.8 A solution $\psi$ satisfies efficiency, anonymity, weak monotonicity, weak covariance and the weak null player out property if and only if it is an egalitarian Shapley value.

Proof It is straightforward to verify that all egalitarian Shapley values satisfy the weak null player out property. Now, suppose that solution $\psi$ satisfies these properties on $\mathcal{G}$. For games on $\mathcal{G}_{3}$ uniqueness follows from Theorem 4.5. For game $(N, v)$ with $|N|=2$, take an $h \in \mathbb{N} \backslash\{N\}$, and consider the game $(N \cup\{h\}, \bar{v})$ given by $\bar{v}(S)=v(S \cap N)$ for all $S \subseteq N \cup\{h\}$. Hence, $h$ is a null player in $(N \cup\{h\}, \bar{v})$. By Theorem 4.5 the payoffs in $(N \cup\{h\}, \bar{v})$ are determined. If $N=\{i, j\}$ then the weak null player out property implies that $\psi_{i}(N \cup\{h\}, \bar{v})-\psi_{i}(N, v)=\psi_{j}(N \cup\{h\}, \bar{v})-\psi_{j}(N, v)$. With efficiency $\psi(N, v)$ is determined.

Note that in the proof of Theorem 4.8 we use the weak null player out property only to determine the payoffs for two player games from three player games. For other games we determine the payoffs by the other axioms as shown in the proof of Theorem 4.5.

We end this section by remarking that the truly interesting aspect behind the above result is that all these simple and natural properties characterize the whole class of egalitarian Shapley values. Hence, balancing between marginalism and egalitarianism is equivalent to the issue how monotonic a solution is preferred to be. This also provides an alternative angle for planners to consider and make social policy.

\section{Implementation}

In the previous two sections we characterized the egalitarian Shapley values from a cooperative viewpoint. Next we study these solutions from a non-cooperative perspective. In the literature various implementations of the Shapley value can be found, see e.g. Gul (1989), Hart and Mas-Colell (1996) and Pérez-Castrillo and Wettstein (2001). In this section we adapt the last mechanism and obtain the following mechanism that implements any egalitarian Shapley value as the unique subgame perfect equilibrium outcome. The connection as well as the difference between our mechanism (implementing egalitarian Shapley values) and others in the literature will be discussed at the end of the section. 
The bidding mechanism is defined recursively as follows. When there is only one player, say $i$, in the game, this player simply gets its stand-alone payoff, $v(\{i\})$. Given the rules of the mechanism for games with at most $k-1<n$ players, the bidding game for a set of $k$ players proceeds in rounds. Dependent upon the strategies of the corresponding players, the bidding game may have up to $n$ rounds, each consisting of four stages. Let $N_{t}$ be the player set of the game with which the bidding game of each round $t \in\{1, \ldots, n\}$ will start.

Round 1: $N_{1}=N$. Go to Stage 1.

Stage 1: Each player $i \in N$ makes bids $b_{j}^{i} \in \mathbb{R}$ for every $j \neq i$. For each $i \in N$, let $B^{i}=\sum_{j \neq i}\left(b_{j}^{i}-b_{i}^{j}\right)$ be the net bid of player $i$ measuring its 'relative' willingness to be the proposer. Let $i_{1}^{*}$ be the player with the highest net bid in this round. (In case of a non-unique maximizer we choose any of these maximal bidders to be the 'winner' with equal probability.) Once the winner $i_{1}^{*}$ has been determined, player $i_{1}^{*}$ pays every other player $j \in N \backslash\left\{i_{1}^{*}\right\}$ its offered bid $b_{j}^{i_{1}^{*}}$. The 'winner' $i_{1}^{*}$ becomes the proposer in the next stage. Go to Stage 2.

Stage 2: Player $i_{1}^{*}$ proposes an offer $y_{j}^{i_{1}^{*}} \in \mathbb{R}$ to every player $j \neq i_{1}^{*}$. (This offer is additional to the bids paid at stage 1.) Go to Stage 3 .

Stage 3: The players other than $i_{1}^{*}$, sequentially, either accept or reject the offer. If at least one player rejects it, then the offer is rejected. Otherwise, the offer is accepted. Go to Stage 4.

Stage 4: If the offer is accepted, then each player $j \in N \backslash\left\{i_{1}^{*}\right\}$ receives $y_{j}^{i_{1}^{*}}$ and player $i_{1}^{*}$ obtains $v(N)-\sum_{j \neq i_{1}^{*}} y_{j}^{i_{1}^{*}}$. Hence, in this case the final payoff to player $j \neq i_{1}^{*}$ is $y_{j}^{i_{1}^{*}}+b_{j}^{i_{1}^{*}}$, while player $i_{1}^{*}$ receives $v(N)-\sum_{j \neq i_{1}^{*}}\left(y_{j}^{i_{1}^{*}}+\right.$ $\left.b_{j}^{i_{1}^{*}}\right)$. Stop.

If the offer is rejected then with probability $\alpha \in[0,1]$ player $i_{1}^{*}$ leaves the game and obtains her stand-alone payoff $v\left(\left\{i_{1}^{*}\right\}\right)$, while the players in $N \backslash\left\{i_{1}^{*}\right\}$ proceed to round 2 to bargain over $v\left(N \backslash\left\{i_{1}^{*}\right\}\right)$. With probability $(1-\alpha) \in[0,1]$ the game breaks down and all players, including the proposer $i_{1}^{*}$, get zero payoffs at this stage (and thus only the bids of stage 1 are transferred, i.e. the payoff to player $j \neq i_{1}^{*}$ is $b_{j}^{i_{1}^{*}}$ and the payoff to the proposer $i_{1}^{*}$ is $-\sum_{j \neq i_{1}^{*}} b_{j}^{i_{1}^{*}}$.). Stop.

In the following rounds, the stages 1, 2 and 3 are the same as in round 1 but with the reduced player set where the proposer in the previous round has left. However, at stage 4 of the following rounds there is no possibility of breakdown. To be complete we describe the following rounds.

Round $t, t \in\{2, \ldots, n-1\}: N_{t}=N_{t-1} \backslash\left\{i_{t-1}^{*}\right\}$. Go to Stage 1 .

Stage 1: Each player $i \in N_{t}$ makes bids $b_{j}^{i} \in \mathbb{R}$ for every $j \neq i$. For each $i \in N_{t}$, let $B^{i}=\sum_{j \in N_{t} \backslash\{i\}}\left(b_{j}^{i}-b_{i}^{j}\right)$, be the net bid of player $i$. 
Let $i_{t}^{*}$ be the player with the highest net bid of round $t$. (In case of a non-unique maximizer we choose any of these maximal bidders to be the 'winner' with equal probability.) Once the winner $i_{t}^{*}$ has been determined, player $i_{t}^{*}$ pays every other player $j \in N_{t} \backslash\left\{i_{t}^{*}\right\}$, its offered bid $b_{j}^{i_{t}^{*}}$. The 'winner' $i_{t}^{*}$ becomes the proposer in the next stage. Go to Stage 2.

Stage 2: Player $i_{t}^{*}$ proposes an offer $y_{j}^{i_{t}^{*}} \in \mathbb{R}$ to every player $j \in N_{t} \backslash\left\{i_{t}^{*}\right\}$. (This offer is additional to the bids paid at stage 1.) Go to Stage 3.

Stage 3: The players other than $i_{t}^{*}$, sequentially, either accept or reject the offer. If at least one player rejects it, then the offer is rejected. Otherwise, the offer is accepted. Go to Stage 4.

Stage 4: If the offer is accepted, then each player $j \in N_{t} \backslash\left\{i_{t}^{*}\right\}$ receives $y_{j}^{i_{t}^{*}}$ and player $i_{t}^{*}$ obtains $v\left(N_{t}\right)-\sum_{j \in N_{t} \backslash\left\{i_{t}^{*}\right\}} y_{j}^{i_{t}^{*}}$ at this stage. Hence, in this case the final payoff to player $j \in N_{t} \backslash\left\{i_{t}^{*}\right\}$ is $y_{j}^{i_{t}^{*}}+b_{j}^{i_{t}^{*}}+\sum_{k=1}^{t-1} b_{j}^{i_{k}^{*}}$, while player $i_{t}^{*}$ receives $v\left(N_{t}\right)-\sum_{j \in N_{t} \backslash\left\{i_{t}^{*}\right\}}\left(y_{j}^{i_{t}^{*}}+b_{j}^{i_{t}^{*}}\right)+\sum_{k=1}^{t-1} b_{i_{t}^{*}}^{i_{*}^{*}}$. Stop.

If the offer is rejected then player $i_{t}^{*}$ leaves the game and obtains its stand-alone payoff $v\left(\left\{i_{t}^{*}\right\}\right)$, while the players in $N_{t} \backslash\left\{i_{t}^{*}\right\}$ proceed to round $t+1$ to bargain over $v\left(N_{t} \backslash\left\{i_{t}^{*}\right\}\right)$.

Round $n$ : $N_{n}=N_{n-1} \backslash\left\{i_{n-1}^{*}\right\}$. Apparently, $N_{n}$ is a singleton coalition so that it is a one-player game in this round. The game immediately stops such that player $i \in N_{n}$ gets its stand-alone payoff $v\left(N_{n}\right)$. So, its final payoff is $v\left(N_{n}\right)+\sum_{k=1}^{n-1} b_{i}^{i^{*}}$.

Note that this bidding mechanism extends that of Pérez-Castrillo and Wettstein (2001) by allowing for the possibility of breakdown after the offer is rejected in the first round. The option of breakdown after the first round negotiation is very realistic. A conventional explanation is due to the modeling of players' (im)patience level. Alternatively, it can also be seen as the uncertainty with respect to the players not knowing each other or any factors that cause the joint negotiation impossible or too costly to proceed (for example because they speak different languages) after the first round. This breakdown occurs with probability $1-\alpha$. In the same spirit, $\alpha$ indicates how likely the game will continue rather than stop after the first rejection. If the game does continue (with probability $\alpha$ ), then the players agreed that they can negotiate, and this possibility of breakdown does not occur anymore. Then the whole game can only be stopped by acceptance of all relevant players in a certain round or rejection in a two-player bidding subgame. A further discussion relating the breakdown and continuing probabilities after the first round to real life situations is provided at the end of this section.

Given the characteristic function $v$, we can calculate the final payoffs of the players who are assumed to be risk neutral in the mechanism. In case of rejection in the first round, the expected final gain of proposer $i_{1}^{*}$ is $\alpha v\left(\left\{i^{*}\right\}\right)-\sum_{j \neq i^{*}} b_{j}^{i^{*}}$, whereas every other player $j \neq i_{1}^{*}$ finally obtains $b_{j}^{i^{*}}$ plus the expected payoff due to the contingent (with probability $\alpha$ ) outcome of the mechanism continuing with player set 
$N \backslash\left\{i_{1}^{*}\right\}$. In case of acceptance of the proposal in the first round, the final gain of $i_{1}^{*}$ is $v(N)-\sum_{j \neq i_{1}^{*}}\left(b_{j}^{i_{1}^{*}}+y_{j}^{i_{1}^{*}}\right)$, whereas the final gain of every player $j \neq i_{1}^{*}$ is $b_{j}^{i_{1}^{*}}+y_{j}^{i_{1}^{*}}$.

Next we generalize the result of Pérez-Castrillo and Wettstein (2001) who showed that for $\alpha=1$ this bidding mechanism implements the Shapley value for zero-monotonic games. A TU-game $(N, v)$ is zero-monotonic if $v(S) \geq v(S \backslash\{i\})+v(\{i\})$ for all $S \subseteq N$ and all $i \in S$. It turns out that for any zero-monotonic game such that the 'grand coalition' earns a nonnegative worth, the given bidding mechanism implements the $\alpha$-egalitarian Shapley values as subgame perfect equilibrium (SPE) outcomes. For $T \subset N$ the restricted game $\left(T, v_{T}\right) \in \mathcal{G}$ is given by $v_{T}(S)=v(S)$ for all $S \subseteq T$.

Theorem 5.1 Let $\alpha \in[0,1]$ be the probability that the bidding continues after rejection in the first round, and let $v \in \mathcal{G}$ be a zero monotonic game with $v(N) \geq 0$. Then the outcome in any subgame perfect equilibrium of the bidding mechanism coincides with the payoff vector $\varphi^{\alpha}(N, v)$.

$\operatorname{Proof}$ Let $(N, v)$ be a zero-monotonic game with $v(N) \geq 0$, and let $\alpha \in[0,1]$. The proof can be readily constructed by observing the fact that playing the game $(N, v)$ by the bidding mechanism defined above is 'effectively equivalent' to playing the modified game $(N, \alpha v+(1-\alpha) f(v))$ by the bidding mechanism defined by Pérez-Castrillo and Wettstein (2001). ${ }^{11}$ Then, by Theorem 1 in Pérez-Castrillo and Wettstein (2001), it follows that the SPE outcome of the current mechanism is the Shapley value of the game $\alpha v+(1-\alpha) f(v)$, which is $\varphi^{\alpha}(N, v)$.

We like to note that an intriguing implication of the above mechanism and the implementation result is that the equilibrium outcome is closer to be egalitarian if the negotiation breakdown occurs more likely, whereas it is closer to be marginalistic if the negotiation continues. This is fairly consistent to our real life observation. Usually, when negotiating parties are sufficiently patient, a more competitive outcome is expected. However, if the time limit is stringent but the cooperative gain is too lucrative to resist, then a more egalitarian rule could be adopted straight away with smooth consensus.

It may be worth discussing the relationship between our mechanism and others in the literature. Actually, only few works address the issue of implementing mixed solutions like the egalitarian Shapley values. Hart and Mas-Colell (1996) discussed a generalized version of their multilateral bargaining game and found that, for some $\theta \in(0,1)$, if the proposer drops out with probability $(1-\rho) \theta$, whereas each responder drops out with equal probability $(1-\rho)(1-\theta) /(s-1),{ }^{12}$ then the game implements the corresponding egalitarian Shapley value with respect to $\theta$ in stationary subgame perfect equilibria. However, we like to point out that this result only obtains the egalitarian Shapley value as the equilibrium outcome in expected terms rather than in actual terms, as the game requires an ex ante probability distribution for players to be proposer. Moreover, the result relies on the use of two parameters. By contrast,

\footnotetext{
11 The detailed and complete proof can be obtained from the authors.

12 Here in the bargaining model of Hart and Mas-Colell (1996), $\rho$ denotes the probability that the game will continue with the same set of active players $S$, i.e., a case where no player drops out, and $s$ denotes the cardinality of the set of active players.
} 
the mechanism introduced above implements the value in actual terms, and uses only one parameter $\alpha$ denoting the probability that the game will continue. Moreover, this parameter only takes effect once: when the proposal is rejected in the first round. If the game actually enters the next round, then no breakdown happens and thus no parameter will appear thereafter. In this sense, the mechanism of the paper seems to provide a more clear-cut result and can help to illustrate the relationship between bargaining equilibrium and negotiation breakdown probability more directly.

In Ju and Wettstein (2009), a renegotiation mechanism is briefly suggested where the rejected proposer can renegotiate with the accepted proposer of the remaining players. If no deal is reached in the renegotiation then the previously rejected proposer will be "finally rejected" and get zero payoff. This mechanism implements the egalitarian Shapley value corresponding to the probability about who has the right to make the offer in renegotiation. Hence, the result in Ju and Wettstein (2009) discovers the relationship between equilibrium outcome and power to offer in renegotiation, whereas our mechanism focusses on a breakdown scenario in bargaining.

We end this section by remarking that the design of the negotiation game and the corresponding implementation result model features that are relevant for many real life negotiations. In a multilateral bargaining situation we often see that discussion may completely stop when an initial proposal is found unacceptable and immediately turned down by some parties. On the other hand, if the parties agree on the basic framework, then a detailed arrangement can be finally made through many rounds of subsequent negotiations. For example, the Bali Roadmap basically makes the nations agree to continue for further detailed negotiations, and after this 'first round' the game enters a no breakdown subsequent two-year process towards the 2009 United Nations Climate Change Summit in Copenhagen (despite the not so satisfying deal made so far). An alternative example is the joint pay negotiation among several unions and employers in a sector. Sometimes we do see that negotiation immediately breaks down. But, when the parties went through the very difficult first round, negotiation usually moves on to a settlement, although it may take some time. This paper provides a basic mechanism which can serve as a reference point for modeling and analyzing such negotiations. Many variations in real practice could lead to interesting studies, such as taking account of outside options and even retaliation strategies in multilateral negotiation.

\section{Alternative characterizations and conclusion}

In this paper we formulated the trade-off between marginalism and egalitarianism in cooperative games by considering convex combinations of the Shapley value and the equal division solution. We provided three main characterizations of these egalitarian Shapley values by consistency, monotonicity (both cooperative) and implementation (non-cooperative). Since these important types of properties were shared in common by these solutions, we consider the egalitarian Shapley values as an important concept to make the trade-off between marginalism and egalitarianism.

Other axiomatizations of the Shapley value can or have been generalized for egalitarian Shapley values. For example, it is obvious that $\varphi^{\alpha}$ is characterized by efficiency, 
symmetry, linearity and, instead of the null player property, requiring the $\alpha$-egalitarian null player property stating that $\psi_{i}(N, v)=(1-\alpha) \frac{v(N)}{n}, \alpha \in[0,1]$, for every null player $i$ in game $(N, v)$. This is shown by Joosten (1996) who also provides axiomatizations using a modified balanced contribution property (Myerson 1980), a modified potential and a modified reduced game consistency (Hart and Mas-Colell 1989).

Also, we can generalize the recursive formula of Maschler and Owen (1989), yielding

$$
\begin{aligned}
\varphi_{i}^{\alpha}(N, v)= & \frac{1}{n} \sum_{j \neq i} \varphi_{i}^{\alpha}\left(N \backslash\{j\}, v_{N \backslash\{j\}}\right) \\
& +\frac{1}{n}\left(v(N)-\alpha v(N \backslash\{i\})-(1-\alpha) \frac{\sum_{j \neq i} v(N \backslash\{j\})}{n-1}\right), \text { for all } i \in N .
\end{aligned}
$$

Taking $\alpha=1$ yields the recursive formula of the Shapley value given in Maschler and Owen (1989).

Malawski (2004) obtains the egalitarian Shapley values by a procedure where for every order of entrance to the 'grand coalition' player $i \in N$ gets a share $\alpha$ in its marginal contribution and the predecessors of $i$ equally share the remainder of $i$ 's marginal contribution. ${ }^{13}$ Taking the average over all orders of entrance yields the corresponding $\alpha$-egalitarian Shapley value as expected payoffs. This heuristic approach can be viewed as a procedural interpretation on how agents take their social responsibilities in an egalitarian sense (like volunteer, donation, charity, etc.) while their contributions are remunerated on a marginalistic basis. Hence, it further shows that the egalitarian Shapley values provide a measure of balancing marginalism and egalitarianism. In a similar spirit, Ju et al. (2007) allocate for every order of entrance the surplus $v(S)-v(S \backslash\{i\})-v(\{i\})$ instead of the marginal contribution among the entrant $i$ and its predecessor, and assign the worth $v(\{i\})$ fully to player $i$. This yields the convex combinations of the Shapley value and the CIS-value ${ }^{14}$, the so-called generalized consensus values, as expected payoffs. For characterizations and implementations of generalized consensus values, we refer to Ju et al. (2007) and Ju and Wettstein (2009).

The CIS-value is an egalitarian solution which is computed above the disagreement point (i.e., players' stand-alone payoffs) when the game is superadditive. Although the generalized consensus values may look quite similar to the egalitarian Shapley values, their axiomatic characterizations are rather different. For example, all generalized consensus values satisfy standardness for two-player games, but the Shapley value is the only generalized consensus value satisfying Sobolev (1973)'s reduced game consistency. On the other hand, in this paper we showed that all egalitarian Shapley values satisfy Sobolev's reduced game consistency, but the Shapley value is the only 'standard' solution in this class. We characterized all egalitarian Shapley values using a paramatrized standardness.

\footnotetext{
13 We remark that Malawski's result is more general than just considering this procedure.

14 The CIS-value (see Driessen and Funaki 1991), also called the equal surplus solution, is given by $C I S_{i}(N, v)=v(\{i\})+\frac{v(N)-\sum_{j \in N} v(\{j\})}{n}$ for all $i \in N$.
} 
As mentioned at the end of Sect. 3, Joosten (1996) axiomatizes the class of egalitarian Shapley values by a parametrized standardness for two-player games, and a paramatrized Hart and Mas-Colell reduced game consistency. The main disadvantage thus is that both axioms are paramatrized, while in our axiomatization all egalitarian Shapley values satisfy the same Sobolev reduced game consistency, and differ only in the specific $\alpha$-standardness for two player games that is applied.

Axiomatic comparisons between various equal division solutions have been made by van den Brink and Funaki (2009) who consider a class of equal surplus sharing solutions that includes the equal division solution, the CIS-value, the ENSC-value (i.e. the dual of the CIS-value) and all their convex combinations. Further generalizations of the egalitarian Shapley values and generalized consensus values consider convex combinations of any of these equal surplus sharing solutions with the Shapley value (or even the more general semi-values, see Dubey et al. (1981)).

Acknowledgements The authors thank associate editor and a referee for useful comments on a previous version of the paper. Yukihiko Funaki thanks the financial support from the Netherlands Organization for Scientific Research, grant B 45-299. Yuan Ju thanks the financial support of the Anniversary Lectureship granted by the University of York.

Open Access This article is distributed under the terms of the Creative Commons Attribution Noncommercial License which permits any noncommercial use, distribution, and reproduction in any medium, provided the original author(s) and source are credited.

\section{References}

Ambec S, Sprumont Y (2002) Sharing a river. J Econ Theory 107:453-462

Curiel I, Pederzoli G, Tijs S (1989) Sequencing games. Eur J Oper Res 40:344-351

Derks JJM, Haller HH (1999) Null players out? linear values for games with variable supports. Int Game Theory Rev 1:301-314

Driessen TSH (1991) A survey of consistency properties in cooperative game theory. SIAM Rev 33:43-59

Driessen TSH, Funaki Y (1991) Coincidence of and collinearity between game theoretic solutions. OR Spektrum 13:15-30

Dubey P, Neyman A, Weber RJ (1981) Value theory without efficiency. Mat Oper Res 6:122-128

Dutta B, Ray D (1989) A concept of egalitarianism under participation constraints. Econometrica 57: $615-635$

Gul F (1989) Bargaining foundations of shapely value. Econometrica 57:81-95

Graham DA, Marshall RC, Richard JF (1990) Differential payments within a bidder coalition and the Shapley value. Am Econ Rev 80:493-510

Harsanyi JC (1959) A bargaining model for cooperative n-person games. In: Tucker AW, Luce RD (eds) Contributions to the theory of games IV. Princeton UP, Princeton pp 325-355

Hart S, Mas-Colell A (1988) The potential of the Shapley value. In: Roth AE (ed) The Shapley value. Essays in Honor of L.S. Shapley. Cambridge University Press, pp 127-137

Hart S, Mas-Colell A (1989) Potential, value and consistency. Econometrica 57:589-614

Hart S, Mas-Colell A (1996) Bargaining and value. Econometrica 64:357-380

Joosten R (1996) Dynamics, equilibria and values dissertation. Maastricht University

Ju Y, Wettstein D (2009) Implementing cooperative solution concepts: a generalized bidding approach. Econ Theory 39:307-330

Ju Y, Borm PEM, Ruys PHM (2007) The consensus value : a new solution concept for cooperative games. Soc Choice Welf 28:685-703

Littlechild SC, Owen G (1973) A simple expression for the Shapley value in a special case. Manag Sci 20:370-372 
Malawski M (2004) Procedural values for cooperative games. IPI PAN Report 982, Instytut Podstaw Informatyki PAN, Warsaw

Maniquet F (2003) A characterization of the Shapley value in queueing problems. J Econ Theory 109: 90-103

Maschler M, Owen G (1989) The consistent Shapley value for hyperplane games. Int J Game Theory 18:389-407

Myerson RB (1980) Conference structures and fair allocation rules. Int J Game Theory 9:169-182

Ni D, Wang Y (2007) Sharing a polluted river. Games Econ Behav 60:176-186

Pérez-Castrillo D, Wettstein D (2001) Bidding for the surplus: a non-cooperative approach to the Shapley value. J Econ Theory 100:274-294

Salles M (2008) Limited rights as partial Veto and Sen's impossibility theorem. In: Pattanaik PK, Tadenuma $\mathrm{K}, \mathrm{Xu}$ Y, Yoshihara N (eds) Rational choice and social welfare. Springer, pp 11-23

Sen A (1970) The impossibility of a paretian liberal. J Polit Econ 78:152-157

Shapley LS (1953) A value for $n$-person games. In: Kuhn HW, Tucker AW (eds) Annals of mathematics studies 28 (contributions to the theory of games, vol 2). Princeton University Press, Princeton pp 307-317

Sobolev AI (1973) The functional equations that give the payoffs of the players in an $N$-person game. In: Vilkas E (ed) Advaces in game theory. Izdat "Mintis", Vilnius, pp 151-153 (in Russion)

van den Brink R (2001) An axiomatization of the Shapley value using a fairness property. Int J Game Theory 30:309-319

van den Brink R (2007) Null or nullifying players: the difference between the Shapley value and equal division solutions. J Econ Theory 136:767-775

van den Brink R, Funaki Y (2009) Axiomatizations of a class of equal surplus sharing solutions for cooperative games with transferable utility. Theory Decis 67:303-340

Yanovskaya E, Driessen TSH (2002) Note on linear consistency of anonymous values for TU-games. Int J Game Theory 30:601-609

Young HP (1985) Monotonic solutions of cooperative games. Int J Game Theory 14:65-72 